\title{
FDG Uptake of Normal Canine Brain Assessed by High-Resolution Research Tomography-Positron Emission Tomography and 7 T-Magnetic Resonance Imaging
}

\author{
Byeong-Teck KANG ${ }^{1}$, Young-Don SON ${ }^{2}$, Sang-Rae LEE ${ }^{3)}$, Dong-In JUNG ${ }^{4}$, Dong-Eog $\mathrm{KIM}^{5)}$, Kyu-Tae CHANG ${ }^{3)}$, \\ Zang-Hee $\mathrm{CHO}^{2)}$ and Hee-Myung PARK ${ }^{6} *$ \\ 1) Laboratory of Veterinary Dermatology and Neurology, College of Veterinary Medicine, Chungbuk National University, Cheongju, \\ Chungbuk 361-763, South Korea \\ 2)Neuroscience Research Institute, Gachon University of Medicine and Science, Incheon 405-760, South Korea \\ 3) The National Primate Research Center, Korea Research Institute of Bioscience and Biotechnology (KRIBB), Ochang, Chungbuk \\ 363-883, South Korea \\ 4) Research Institute of Life Sciences, Gyeongsang National University, Jinju 660-701, South Korea \\ ${ }^{5)}$ Molecular Imaging and Neurovascular Research (MINER) Laboratory, Dongguk University Ilsan Hospital, Goyang 410-773, South \\ Korea \\ 6) Department of Veterinary Internal Medicine, College of Veterinary Medicine, Konkuk University, Seoul 143-701, South Korea
}

(Received 7 March 2012/Accepted 2 May 2012/Published online in J-STAGE 22 May 2012)

ABSTRACT. The purpose of this study was to assess the normal distribution of ${ }^{18} \mathrm{~F}$-fluoro-2-deoxy-D-glucose (FDG) uptake of canine brain structures using a high-resolution research tomography-positron emission tomography (HRRT-PET) and 7 T-magnetic resonance imaging (MRI) fusion system. FDG-PET and T2-weighted MRI of the brain were performed on 4 healthy laboratory beagle dogs. On MRI, regions of interests (ROIs) were manually drawn over 51 intracranial structures, including nine gross structures and 42 detailed structures. Relative standard uptake value ratio (rSUV=SUV of ROI/SUV of whole brain) was calculated for each ROI. The HRRT-PET and 7 T-MRI fusion imaging system demonstrated significant differences in glucose metabolism among various intracranial structures. Among gross structures, the midbrain and the pons and medulla oblongata had the highest uptake (rSUV: $1.12 \pm 0.03$ ) and lowest uptake (rSUV: 0.90 $\pm 0.06)$ of FDG, respectively. When rSUVs were calculated on detailed regions, the caudal colliculus and the longitudinal fibers of pons had the highest (rSUV: $1.62 \pm 0.05$ ) and the lowest (rSUV: $0.63 \pm 0.03$ ) glucose metabolism, respectively. Because the high resolution of PET-MRI fusion images provided clearly identifiable metabolic activities of canine brain, the HRRT-PET and 7 T-MRI fusion imaging might be a good tool for evaluation of intracranial diseases in canines.

KEY WORDS: 7 T-MRI, canine brain, FDG, HRRT-PET.

doi: 10.1292/jvms.12-0107; J. Vet. Med. Sci. 74(10): 1261-1267, 2012

Positron emission tomography (PET) is a nuclear medical technique that enables qualitative and quantitative measurements of tissue metabolism and physiology in vivo with positron-emitting radionucleotides [29]. Compared with computed tomography (CT) and magnetic resonance imaging (MRI), PET images rarely represent the accurate anatomical information of the brain due to the low spatial resolution [32]. This resolution limit of the PET has been complemented by combining the PET images with the CT images [22]. However, CT has a number of shortcomings in brain imaging, such as the poor contrast of soft tissue and hazardous X-ray radiation. Therefore, MRI was sought as an alternative to $\mathrm{CT}[6]$.

High-resolution research tomography (HRRT)-PET has the highest resolution among the PET systems developed to date [6]. 7 T-MRI can visualize fine structures of the brain, which are difficult to unequivocally identify at

*Correspondence to: Park, H.-M., Department of Veterinary Internal Medicine, Konkuk University, \#1 Hwayang-dong, Gwangjin-gu, Seoul 143-701, South Korea.

e-mail: parkhee@konkuk.ac.kr

(C)2012 The Japanese Society of Veterinary Science lower magnetic field [3]. Until recently, several attempts to combine the high resolution imaging capability of 7 T-MRI with HRRT-PET have been tried in humans and non-human primates $[4,5,12,21,31]$, but not in canines. Previously, 7 T-MRI provided good spatial and contrast resolution for the identification of clinically relevant brain anatomy in dogs $[18,27]$. Therefore, we thought that the HRRT-PET and 7 T-MRI fusion system could be used for the evaluation of canine brain in clinical and research purposes because of its highest sensitivity in both molecular and anatomical imaging of the brain.

Numerous radioactive labeled compounds, ligands, and probes have been developed for PET application [6]. Among them, the glucose analog ${ }^{18} \mathrm{~F}$-fluoro-2-deoxy-D-glucose (FDG) is the major radionuclide in PET imaging. FDG accumulation reflects the rate of glucose utilization in a tissue, and mapping of FDG uptake within the body is altered in various inflammatory and neoplastic diseases [16]. Therefore, FDG-PET has assumed an increasingly important role in clinics and neurosciences by detecting the alteration of glucose utilization shown in various intracranial diseases $[10,13,15,25,28]$. Recently, FDG-PET has become available in veterinary medicine to obtain metabolic information 
of intracranial inflammation and tumor $[8,17,19]$.

Although some previous studies describing normal glucose uptake of canine brain have reported comparisons with suspected or confirmed disease [11, 14, 24], all data were obtained from gross structures by the PET/CT or middlefield MRI (1.5 T). Therefore, the purpose of this study was to assess FDG uptake of normal canine brain according to detailed structures using a HRRT-PET and 7 T-MRI fusion imaging system.

\section{MATERIALS AND METHODS}

Animals: The study population comprised four healthy laboratory beagles (two males and two females, 5-6-yearold, weighing 12-14 kg; Harlan Interfauna, Huntingdon, Cambridgeshire, U.K.) were used. All of the dogs were healthy without a history of neurologic disease; they had no signs of neurologic problems on physical and neurological examinations. They were screened for metabolic diseases by means of a complete blood count and serum chemistry analysis. After arrival at our facility, the beagles were acclimated and assessed daily for neurobehavioral abnormalities and general health status for 2 weeks. Each dog was housed under a controlled photoperiod (lights on, 08:00-20:00) in a single cage and fed twice daily with commercial dry food (Science Diet; Hill's Pet Nutrition, Topeka, KS, U.S.A.), and fresh water was supplied continuously by automatic dispensers. Room temperature was $18-24^{\circ} \mathrm{C}$, with a relative humidity of $55 \pm 10 \%$ and eight air changes per hour. The quarantine and housing measures for the dogs were approved by the Institutional Animal Care and Use Committee of Konkuk University (Seoul, South Korea), which also approved the experimental protocol.

Animal preparation and anesthesia for imaging: All dogs were fasted for $12 \mathrm{hr}$ and were confirmed to have a normal blood glucose level prior to imaging. FDG was produced immediately before injection using an onsite cyclotron (Eclipse HP Cyclotron; CTI Molecular Imaging, Knoxville, TN, U.S.A.) at the Neuroscience Research Institute of Gachon University (Incheon, South Korea). Five minutes after premedication with medetomidine (30 $\mu \mathrm{g} / \mathrm{kg}$ IM; Domitor; Pfizer, Seoul, South Korea), a bolus injection of FDG (0.4 $\mathrm{mCi} / \mathrm{kg}$ ) was given through a cephalic venous catheter for $30 \mathrm{sec}$ and then the catheter was flushed immediately with $10 \mathrm{~m} l$ of normal saline. The dogs were kept caged for $1 \mathrm{hr}$ in a quiet and radioprotective area to minimize movement and ensure a stable FDG uptake. General anesthesia was induced and maintained by a two-time injection of pentobarbital (10 mg/kg IV; Entobal; Hanlim, Gyeonggi, South Korea), oral intubated was introduced, and the dog breathed room air spontaneously. The dog was placed on a shuttle bed, which was used to physically combine the imaging coordinates between the PET and MRI systems [6]. The total duration of anesthesia was $1 \mathrm{hr}$, and vital signs were continuously monitored by end-tidal $\mathrm{CO}_{2}$, heart rate, and oxygen saturation $\left(\mathrm{SPO}_{2}\right)$ using a capnograph and pulse oximeter (Surgivet, Waukesha, WI, U.S.A.). Body temperature was monitored and maintained at $38^{\circ} \mathrm{C}$ using a thermostatically controlled heating pad (Gaymar Industries Inc., Orchard Park, NY, U.S.A.).

Imaging protocol: The FDG-PET scan was conducted for $30 \mathrm{~min}$ on the HRRT (resolution $2.5 \mathrm{~mm}$ full width at half maximum resolution in three-dimensional (3D) acquisition mode; ECAT HRRT; Siemens, Knoxville, TN, U.S.A.), which has a transaxial diameter of $46.9 \mathrm{~cm}$ and an axial field of view (FOV) of $25.2 \mathrm{~cm}$. After the PET scanning, the shuttle system transported the dog to the MRI scanner, and then gradient-echo T2-weighted images of the brain were obtained in the transverse plane for $20 \mathrm{~min}$ by $7 \mathrm{~T}-\mathrm{MRI}$ (a spatial resolution down to 200-300 $\mu \mathrm{m}$; Magnetom 7T; Siemens, Berlin, Germany) with the following parameters: repetition time of $500 \mathrm{msec}$, echo time of $17 \mathrm{msec}, 15 \mathrm{sec}-$ tions, $1.5 \mathrm{~mm}$ slice thickness, $3 \mathrm{~mm}$ interslice gap, FOV of $119 \times 119 \mathrm{~mm}^{2}$, and $576 \times 576$ matrix. PET images were reconstructed using the $3 \mathrm{D}$ ordinary Poisson ordered-subset expectation maximization algorithm with six iterations, 16 subsets, $256 \times 256 \times 207$ matrix, and a pixel size of $1.22 \times$ $1.22 \times 1.22 \mathrm{~mm}^{3}$.

Image analysis: Acquired PET and MR images were automatically co-registered by Vinci 3.80 image analysis software (Max-Planck-Institute, Cologne, Germany). Regions of interests (ROIs) were manually drawn over 51 intracranial structures, including nine gross structures (Fig.1 and Table 1 ; 1 . whole brain, 2. telencephalon, 3. cerebral cortex, 4. cerebral white matter, 5. ventricles, 6. diencephalon, 7. midbrain, 8. cerebellum, 9. pons and medulla oblongata) and 42 detailed structures (Table 2) on 11 consecutive transverse MR images using a two-dimensional ROI tool (Vinci 3.80; Max-Planck-Institute). Because of the ambiguity in the border of detailed structures, circular ROIs with diameters ranging from 0.6 and $3 \mathrm{~mm}$ were placed in the central areas of each detailed structure except for seven structures having clear border, such as frontal, occipital, parietal and temporal lobes (Fig. 1), caudate nucleus, hippocampus, and thalamus. The relevant neuroanatomy was identified by referral to anatomy texts, sectioned cadaver heads, and atlases $[1,7$, 9, 18, 26].

Created ROIs were moved to the corresponding area of PET-MR fusion images, and then a standardized uptake value $[\mathrm{SUV}=$ average tissue concentration of $\mathrm{FDG}(\mathrm{kBq} / \mathrm{m} l) /$ total injected dose $(\mathrm{kBq})$ /body weight $(\mathrm{g})$ ] and relative SUV ratio (rSUV=SUV of ROI/SUV of whole brain) were calculated for each ROIs. Because SUVs obtained using different protocols and PET scanners are not directly comparable with the values of the previous studies, rSUV was utilized for quantitative analysis.

Statistical analyses: Results are expressed as mean \pm standard deviation (SD). The rSUV in each brain region was compared within and between each gross structure using the Kruskal-Wallis test. Pair-wise comparisons of each rSUV between two independent structures were completed using the Mann-Whitney U test. Statistical significance was set at a $P$ value $<0.05$. Statistical analyses were performed using SPSS version 12.0 software (SPSS, Chicago, IL, U.S.A.). 

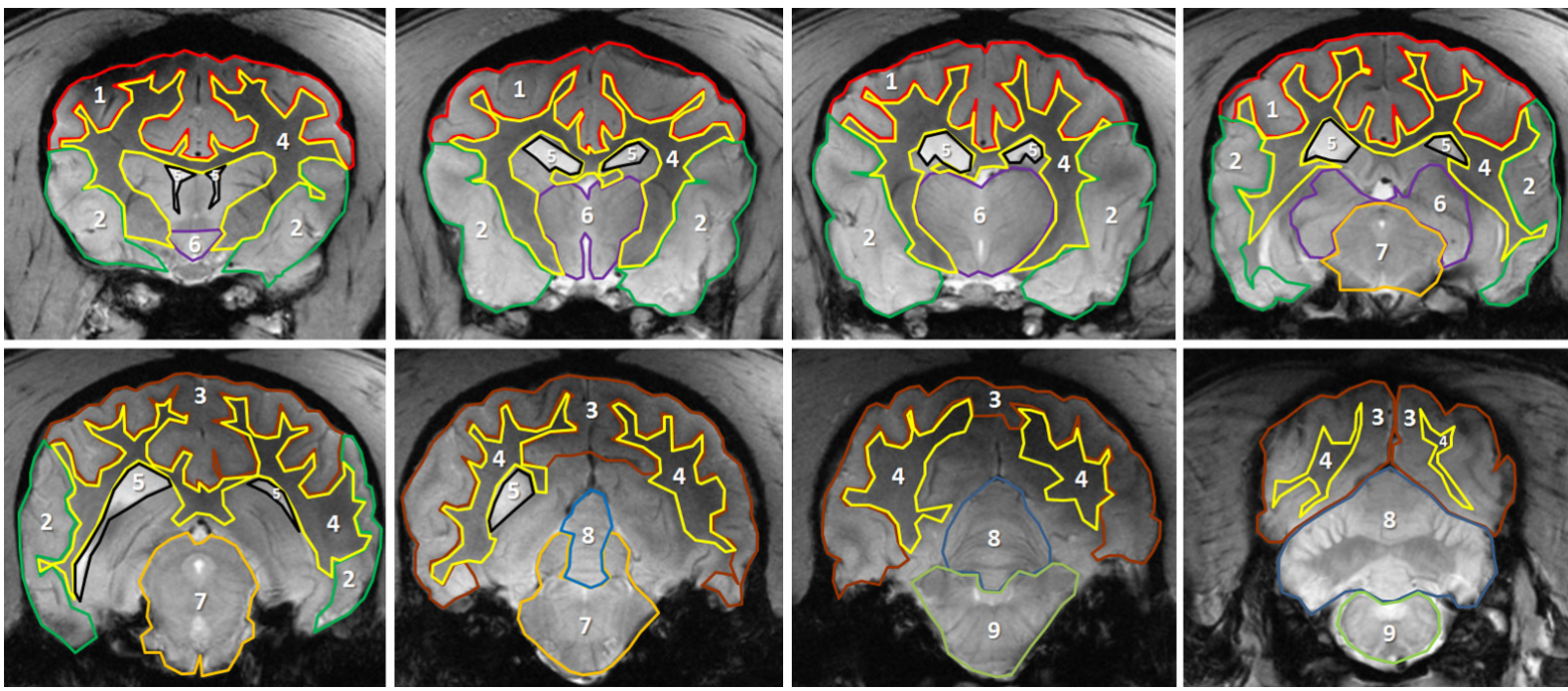

Fig. 1. Representative ROIs for the glucose uptake measurement in seven gross structures [cerebral cortex (1-3), cerebral white matter (4), ventricles (5), diencephalon (6), midbrain (7), cerebellum (8), and pons and medulla oblongata (9)] and three detailed structures [parietal (1), temporal (2) and occipital (3) lobes].
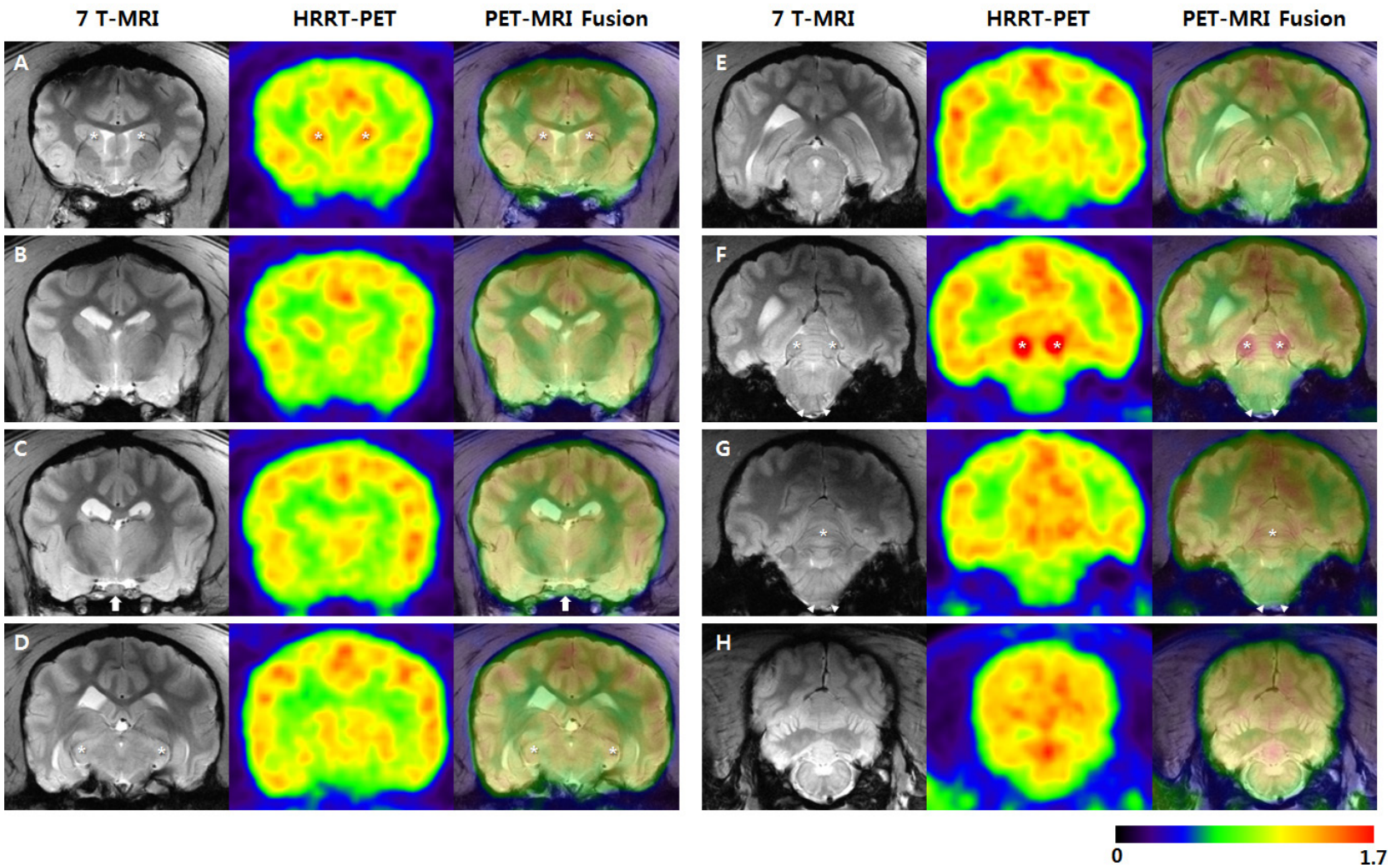

Fig. 2. Representative transverse T2-weighted MRI, FDG-PET, and PET-MRI fusion images at the level of the rostral caudate nucleus (A), piriform lobe/amygdala (B), hypophysis (C), midbrain (D), rostral colliculus (E), caudal colliculus (F), pons (G), and medulla oblongata $(\mathrm{H})$. In PET images, hypermetabolism of glucose is related with reddish to yellowish color, while hypometabolism corresponds to the bluish to greenish color. The highest FDG-uptake was observed in the caudal colliculus (F, *), by contrast, the longitudinal fibers of pons ( $\mathrm{G}$, arrowheads) had the lowest uptake. Glucose metabolism in detailed structures of telencephalon, diencephalon, and cerebellum was the highest in the caudate nucleus $\left(\mathrm{A},{ }^{*}\right)$, the medial geniculate body $\left(\mathrm{D},{ }^{*}\right)$, and the vermis $\left(\mathrm{G},{ }^{*}\right)$, respectively. On the other hand, FDG uptake of detailed structures of telencephalon, diencephalon, and midbrain was the lowest in the cerebral white matter (A to G), the hypophysis (C, arrow), and the crus cerebri (F, arrowheads), respectively. Those hypometabolic areas were clearly identified by combining 7 T-MR images with PET images. The color bar expresses rSUV. 
Table 1. rSUVs and SUVs for gross structures of the canine brain

\begin{tabular}{|c|c|c|}
\hline Location & rSUVs & SUVs \\
\hline Whole brain ${ }^{\text {a) }}$ & $1.00 \pm 0.00$ & $3.72 \pm 0.17$ \\
\hline Telencephalon & $1.00 \pm 0.01$ & $3.71 \pm 0.16$ \\
\hline Cerebral cortex ${ }^{\mathrm{b})}$ & $1.04 \pm 0.02$ & $3.85 \pm 0.08$ \\
\hline Cerebral white matter ${ }^{\mathrm{b}, \mathrm{c})}$ & $0.86 \pm 0.02$ & $3.20 \pm 0.15$ \\
\hline Ventricles $^{\mathrm{b}, \mathrm{c})}$ & $0.76 \pm 0.06$ & $2.83 \pm 0.14$ \\
\hline Diencephalon & $1.03 \pm 0.03$ & $3.83 \pm 0.25$ \\
\hline Midbrain b) & $1.12 \pm 0.03$ & $4.17 \pm 0.23$ \\
\hline Cerebellum ${ }^{\text {b) }}$ & $1.06 \pm 0.01$ & $3.94 \pm 0.16$ \\
\hline Pons and medulla oblongata ${ }^{\mathrm{b}}$ ) & $0.90 \pm 0.06$ & $3.34 \pm 0.35$ \\
\hline
\end{tabular}

The results are expressed as the mean $\pm \mathrm{SD}$. a) $P=0.002$ for difference among five gross structures (telencephalon, diencephalon, midbrain, cerebellum, and pons and medulla oblongata; Kruskal-Wallis test). b) $P<0.02$ for difference in comparison with the whole brain (Mann-Whitney U test). c) $P<0.03$ for difference in comparison with the cerebral cortex (Mann-Whitney U test).

\section{RESULTS}

All dogs were successfully imaged with the PET/MRI scanning procedure, and no adverse side effects were observed. Representative 7 T-MR, HRRT-PET, and PET-MRI fusion images are shown in Fig. 2.

Gross structures: The mean SUV and rSUV and as- sociated SD for nine gross structures are listed in Table 1. Kruskal-Wallis test revealed significant difference of FDG uptakes among five gross structures (1. telencephalon, 2. diencephalon, 3. midbrain, 4. cerebellum, 5. pons and medulla oblongata; $P=0.002$ ). Among those five gross structures, the midbrain had the highest FDG uptake (rSUV: $1.12 \pm 0.03$; Fig. 2D-F). On the other hand, the lowest FDG uptake was observed in the pons and medulla oblongata (rSUV: $0.90 \pm$ 0.06; Fig. $2 \mathrm{G}$ and $2 \mathrm{H}$ ). FDG uptake of the cerebral white matter and the ventricles was significantly lower than that of the cerebral cortex $(P<0.03$; Fig. $2 \mathrm{~A}-\mathrm{G})$.

FDG uptakes of the cerebral cortex, midbrain, and cerebellum were significantly higher than those of the whole brain (rSUV $>1.00, P<0.02$ ). However, glucose metabolisms in the cerebral white matter, ventricles, and pons and medulla oblongata were significantly lower than those in the whole brain $(\mathrm{rSUV}<1.00, P<0.02)$.

Detailed structures: The mean SUV and rSUV and associated SD for 42 detailed structures are listed in Table 2. FDG uptake of each detailed structure was significantly different within each of five gross structures (1. telencephalon, 2. diencephalon, 3. midbrain, 4. cerebellum, 5. pons and medulla oblongata; $P<0.01$ ). Among 42 detailed structures, the highest value was observed in the caudal colliculus (rSUV: 1.62 \pm 0.05 ; Fig. $2 \mathrm{~F}$ ), followed in order by the vermis (rSUV: 1.21 \pm 0.04 ; Fig. $2 \mathrm{G}$ ) and the caudate nucelus (rSUV: $1.20 \pm 0.02$;

Table 2. rSUVs and SUVs for detailed structures of the canine brain

\begin{tabular}{lcc}
\hline \multicolumn{1}{c}{ Location } & rSUVs & SUVs \\
\hline Telencephalon $^{\text {a) }}$ & & \\
Caudate nucleus $^{\text {b) }}$ & $1.20 \pm 0.02$ & $4.45 \pm 0.23$ \\
Cingulate gyrus $^{\text {b) }}$ & $1.12 \pm 0.06$ & $4.14 \pm 0.15$ \\
Frontal lobe $^{\text {b) }}$ & $1.05 \pm 0.01$ & $3.91 \pm 0.15$ \\
Occipital lobe $^{\text {b) }}$ & $1.05 \pm 0.03$ & $3.90 \pm 0.10$ \\
Parietal lobe $^{\text {b) }}$ & $1.04 \pm 0.02$ & $3.86 \pm 0.18$ \\
Rostral commissure $^{\text {b) }}$ & $1.04 \pm 0.02$ & $3.86 \pm 0.13$ \\
Temporal lobe $^{\text {Hippocampus }}$ & $1.00 \pm 0.01$ & $3.73 \pm 0.18$ \\
Septal nuclei $^{\text {Amygdala }}{ }^{\text {b) }}$ & $1.00 \pm 0.08$ & $3.70 \pm 0.17$ \\
Piriform lobe $^{\text {b) }}$ & $0.95 \pm 0.07$ & $3.54 \pm 0.21$ \\
Putamen $^{\text {b) }}$ & $0.93 \pm 0.02$ & $3.47 \pm 0.15$ \\
Internal capsule $^{\text {b) }}$ & $0.89 \pm 0.07$ & $3.33 \pm 0.28$ \\
Corpus callosum $^{\text {b) }}$ & $0.87 \pm 0.00$ & $3.24 \pm 0.15$ \\
\hline Diencephalon $^{\text {a) }}$ & $0.78 \pm 0.04$ & $2.89 \pm 0.20$ \\
Medial geniculate body $^{\text {b) }}$ & $0.76 \pm 0.06$ & $2.81 \pm 0.12$ \\
Lateral geniculate body $^{\text {b) }}$ & $1.16 \pm 0.02$ & $4.30 \pm 0.20$ \\
Thalamus $^{\text {b) }}$ & $1.10 \pm 0.03$ & $4.09 \pm 0.20$ \\
Caudal commissure $^{\text {b) }}$ & $1.09 \pm 0.04$ & $4.06 \pm 0.30$ \\
Hypothalamus $^{\text {Hypophysis }}{ }^{\text {b) }}$ & $1.06 \pm 0.02$ & $3.95 \pm 0.19$ \\
\hline & $1.00 \pm 0.03$ & $3.72 \pm 0.24$ \\
& $0.75 \pm 0.05$ & $2.78 \pm 0.15$ \\
\hline
\end{tabular}

\begin{tabular}{|c|c|c|}
\hline Location & rSUVs & SUVs \\
\hline \multicolumn{3}{|l|}{ Cerebellum $^{\text {a) }}$} \\
\hline Vermis $^{b)}$ & $1.21 \pm 0.04$ & $4.50 \pm 0.35$ \\
\hline Rostral vermis ${ }^{b)}$ & $1.18 \pm 0.04$ & $4.39 \pm 0.20$ \\
\hline Cerebellum ${ }^{\text {b) }}$ & $1.17 \pm 0.02$ & $4.34 \pm 0.21$ \\
\hline Nodulus ${ }^{b)}$ & $1.09 \pm 0.01$ & $4.07 \pm 0.22$ \\
\hline Rostral cerebellar peduncle ${ }^{b}$ ) & $1.09 \pm 0.02$ & $4.04 \pm 0.23$ \\
\hline Cerebellar hemisphere $^{\text {b) }}$ & $1.07 \pm 0.03$ & $3.98 \pm 0.06$ \\
\hline Caudal cerebellar peduncle & $1.04 \pm 0.06$ & $3.87 \pm 0.34$ \\
\hline Fastigeal nucleus & $1.01 \pm 0.10$ & $3.76 \pm 0.51$ \\
\hline Interpositus nucleus & $0.98 \pm 0.12$ & $3.64 \pm 0.54$ \\
\hline Dentate nucleus & $0.97 \pm 0.10$ & $3.60 \pm 0.46$ \\
\hline \multicolumn{3}{|l|}{ Midbrain a) } \\
\hline Caudal colliculus ${ }^{\text {b) }}$ & $1.62 \pm 0.05$ & $6.00 \pm 0.16$ \\
\hline Oculomotor nucleus $^{\text {b) }}$ & $1.11 \pm 0.04$ & $4.12 \pm 0.32$ \\
\hline Periaquedeuctal gray matter ${ }^{b}$ ) & $1.10 \pm 0.06$ & $4.10 \pm 0.31$ \\
\hline Brachium of caudal colliculus ${ }^{\text {b) }}$ & $1.05 \pm 0.02$ & $3.92 \pm 0.22$ \\
\hline Reticular formation & $1.03 \pm 0.04$ & $3.82 \pm 0.26$ \\
\hline Red nucleus & $0.95 \pm 0.10$ & $3.55 \pm 0.51$ \\
\hline Interpeduncular nucleus & $0.85 \pm 0.14$ & $3.19 \pm 0.57$ \\
\hline Substantia nigra ${ }^{b)}$ & $0.80 \pm 0.06$ & $2.98 \pm 0.31$ \\
\hline Crus cerebri b) & $0.79 \pm 0.03$ & $2.92 \pm 0.17$ \\
\hline \multicolumn{3}{|l|}{ Pons and medulla oblongata a) } \\
\hline Vestibular nuclei & $1.04 \pm 0.10$ & $3.90 \pm 0.51$ \\
\hline Middle cerebellar peduncle ${ }^{\text {b) }}$ & $0.83 \pm 0.05$ & $3.11 \pm 0.31$ \\
\hline Longitudinal fibers of pons ${ }^{b}$ ) & $0.63 \pm 0.03$ & $2.36 \pm 0.22$ \\
\hline
\end{tabular}

The results are expressed as the mean $\pm \mathrm{SD}$. a) $P<0.01$ for difference among detailed structures within each five gross structures (Kruskal-Wallis test). b) $P<0.02$ for difference compared with the whole brain (Mann-Whitney U test). 
Fig. 2A). On the other hand, the lowest uptake was noted in the longitudinal fibers of pons (rSUV: $0.63 \pm 0.03$; Fig. $2 \mathrm{G}$ ), followed in order by the hypophysis (rSUV: $0.75 \pm 0.05$; Fig. 2C) and the structures composing cerebral white matter, such as the corpus callosum (rSUV: $0.76 \pm 0.06$; Fig. $2 \mathrm{~A}-\mathrm{E}$ ) and the internal capsule (rSUV: $0.78 \pm 0.04$; Fig. $2 \mathrm{~A}-\mathrm{C}$ ). The anatomical location of those hypometabolic areas was not distinguishable by the PET image alone. By combining the 7 T-MR images, areas with low FDG uptake were more precisely identified.

When the relative metabolic activities of individual detailed structures were considered, glucose metabolisms in six structures of the telencephalon (caudate nucleus, cingulated gyrus, frontal lobe, occipital lobe, parietal lobe, and rostral commissure), four structures of the diencephalon (medial and lateral geniculate bodies, thalamus, and caudal commissure), four structures of the midbrain (caudal colliculus, oculomotor nucleus, periaquedeuctal gray matter, and brachium of caudal colliculus), and six structures of the cerebellum (vermis, rostral vermis, cerebellum, nodulus, rostral cerebellar peduncle, and cerebellar hemisphere) were significantly higher than those in the whole brain (rSUV $>1.00 ; P<0.02)$. On the other hand, FDG uptakes of 10 structures (amygdala, piriform lobe, putamen, internal capsule, corpus callosum, hypophysis, substantia nigra, crus cerebri, middle cerebellar peduncle, and longitudinal fibers of pons) were significantly lower than those of the whole brain $(\mathrm{rSUV}<1.00, P<0.02)$.

\section{DISCUSSION}

The HRRT-PET and 7 T-MRI fusion imaging system demonstrated significant differences in glucose metabolism among various intracranial structures. Even though the highresolution capability of the HRRT improved the accuracy of SUV measurement for each structure by reducing the partial-volume effect, the PET image alone could not provide accurate anatomical information of hypometabolic areas. Because 7 T MRI clearly visualized canine brain structures by high spatial and contrast resolution, delineation of those areas with low FDG uptake was possible by combining HRRT-PET images with 7 T MRI images.

The significant difference of FDG-uptake among gross brain structures has been reported in dogs [11, 14, 24], and it was comparable with the present findings. However, the distribution of regional glucose uptake differed between the present and previous studies. Previous studies showed that the brain stem has the lowest FDG uptake among gross structures $[11,24]$. Using high resolution of PET-MRI fusion images, we could measure glucose metabolism in separate substructures (diencephalon, midbrain, pons, and medulla oblongata) of the brain stem. The highest FDG-uptake among gross structures was observed in the midbrain, and it was significantly higher than that of the cerebral cortex and the whole brain. On the other hand, the pons and medulla oblongata was the sole substructure of the brain stem having relatively low glucose metabolism in comparison with the whole brain. Therefore, glucose metabolism of the brain stem varied according to the substructures.
Similar to previous studies [11, 24], the highest FDG uptake among substructures of the cerebral cortex was observed in the frontal and occipital lobes, but there were little differences of SUVs between individual cortical regions. Because delineation of the white matter from the cortex is difficult on the PET or CT images, quantification of glucose metabolism has not been performed in the cerebral white matter of dogs. Using high resolution 7 T MRI images, FDG uptake was separately estimated in the cerebral white matter and the ventricles. Glucose uptake of neurons correlates positively with neuronal activity and cerebral blood flow [30]. The cerebral white matter is composed of bundles of myelinated nerve cell processes and does not contain nerve cell bodies. Therefore, glucose metabolism in the cerebral white matter and the ventricles was significantly lower than that in the cerebral cortex. In the cats, blood flow in gray matter was generally 3-4 times that in white matter [30]. Because cerebral blood flow was not measured in this study, direct comparison of blood flow between gray matter and white matter was not possible.

The present study showed metabolic activities of individual detailed structures as well as gross structures. Similar to the gross structures, glucose metabolism of the detailed structures was significantly different within each of gross structures. The caudal colliculus had the highest FDG uptake among 42 detailed structures, and this finding was similar to the result of a previous study [14]. In addition, the highest rate of blood flow was observed in the caudal colliculus of cats [30]. Because the caudal colliculus functions as an auditory reflex center by receiving bilateral cochlear input via the lateral lemniscus, auditory stimulation supplied during the periods of FDG uptake and PET scanning may increase the activity of the caudal colliculus. To minimize the influence of auditory stimulation, a soundproof facility may be needed for PET scanning in the dogs. In contrast, the detailed structures mainly composing of nerve fibers, such as the longitudinal fibers of pons, corpus callosum, intercapsule, crus cerebri, and middle cerebellar penducle, had low FDG uptake due to the deficiency of nerve cell bodies and blood supply.

PET images alone could not distinguish the brain stem related structures in detail. However, the increased spatial resolution at $7 \mathrm{~T}$ MRI led to better delineation and conspicuity of the brain stem and cerebellar nuclei including interpeduncular nucleus, fastigeal nucleus, interpositus nucleus, dentate nucleus, and vestibular nuclei. FDG uptake of those structures was not different with the whole brain. Because brain stem-related structures were easily identified on $7 \mathrm{~T}$ MRI images, the HRRT-PET and 7 T-MRI fusion imaging may be a good tool for evaluation of brain stem disorders in the dogs.

In the previous canine PET studies, ROIs were drawn on the largest area of one or two images only [11, 14, 24]. This method could not reflect glucose metabolism of large area exactly. Thus, FDG uptake of gross structures in this study was evaluated by drawing ROIs on 2-5 consecutive transverse images. Numerous factors, such as size and placement of ROI, anesthesia, scanner specifications, at- 
tenuation correction and reconstruction methods, and time of measurement after radiopharmaceutical injection, may influence brain activity and FDG uptake $[2,20]$. Therefore, SUVs obtained using different protocols and instruments are not directly comparable, and all of the factors require strict standardization when SUVs are compared between different PET studies [23]. For comparison with canine PET studies, we used rSUVs as an alternative method of quantitative analysis.

To minimize body movement, anesthesia is necessary for canine diagnostic imaging, and different anesthetic protocols could result in different whole brain glucose metabolism of the dogs [24]. In the present study, long-acting barbiturate, pentobarbital, was used to maintain anesthesia during imaging and transportation of the dogs, because the shuttle bed was designed to rotate at the middle $180^{\circ}$ clockwise between PET and MRI. The previous canine PET-CT study demonstrated that the intracranial glucose uptake was not significantly different between the dogs receiving medetomidine/ pentobarbital and propofol/isoflurane [24]. On the other hand, medetomidine/tiletamine/zolazepam significantly increased glucose uptake in the whole brain compared with propofol/isoflurane [24]. The influence of anesthetic protocols on intracranial glucose uptake should be evaluated in the HRRT-PET and 7 T-MRI fusion imaging system.

Because gradient-echo imaging has the problem of field nonhomogeneity, T1-weighted imaging is suitable for fusion with PET. However, identification of the brain stem structures and distinction between the cerebral cortex and the white matter were clearer in gradient-echo images than T1-weighted images. In the previous human studies, 2D gradient-echo imaging was utilized for the fusion of HRRT-PET and 7 T-MRI $[5,31]$. Therefore, we used transverse plane of gradient-echo images for the PET-MRI fusion, because of the convenience when identifying relevant neuroanatomy in MR images.

This study demonstrates for the first time the different glucose metabolism among various intracranial structures of normal dogs using a HRRT-PET and 7 T-MRI fusion imaging system. Because the high resolution of PET-MRI fusion images provided clearly identifiable metabolic activities of canine brain, the data acquired from normal dogs might help in clinical and experimental neurology to investigate various intracranial diseases of the dogs such as inflammation, neoplasm, and behavioral disorders.

ACKNOWLEDGMENT. This paper was supported by the SMART Research Professor Program of Konkuk University.

\section{REFERENCES}

1. Assheuer, J. and Sager, M. 1997. MRI and CT Atlas of the Dog, Iowa State University Press, Ames.

2. Boellaard, R. 2009. Standards for PET image acquisition and quantitative data analysis. J. Nucl. Med. 50: 11S-20S. [Medline] [CrossRef]

3. Cho, Z. H. 2009. 7.0 Tesla MRI Brain Atlas: In Vivo Atlas with Cryomacrotome Correlation, Springer, New York.
4. Cho, Z. H., Son, Y. D., Kim, H. K., Kim, N. B., Choi, E. J., Lee, S. Y., Chi, J. G., Park, C. W., Kim, Y. B. and Ogawa, S. 2011. Observation of glucose metabolism in the thalamic nuclei by fusion PET/MRI. J. Nucl. Med. 52: 401-404. [Medline] [CrossRef]

5. Cho, Z. H., Son, Y. D., Kim, H. K., Kim, S. T., Lee, S. Y., Chi, J. G., Park, C. W. and Kim, Y. B. 2010. Substructural hippocampal glucose metabolism observed on PET/MRI. J. Nucl. Med. 51: 1545-1548. [Medline] [CrossRef]

6. Cho, Z. H., Son, Y. D., Kim, H. K., Kim, K. N., Oh, S. H., Han, J. Y., Hong, I. K. and Kim, Y. B. 2008. A fusion PET-MRI system with a high-resolution research tomograph-PET and ultra-high field 7.0 T-MRI for the molecular-genetic imaging of the brain. Proteomics 8: 1302-1323. [Medline] [CrossRef]

7. De Lahunta, A. and Glass, E. 2009. Neuroanatomy by dissection. pp. 6-22. In: Veterinary Neuroanatomy and Clinical Neurology, 3rd ed., Saunders, St. Louis.

8. Eom, K. D., Lim, C. Y., Gu, S. H., Kang, B. T., Kim, Y. B., Jang, D. P., Woo, E. J., Kim, D., Cho, Z. H. and Park, H. M. 2008. Positron emission tomography features of canine necrotizing meningoencephalitis. Vet. Radiol. Ultrasound 49: 595-599. [Medline] [CrossRef]

9. Evans, H. E. 1993. Miller's Anatomy of the Dog, 3rd ed., Saunders, Philadelphia.

10. Fiorella, D. J., Provenzale, J. M., Coleman, R. E., Crain, B. J. and Al-Sugair, A. A. 2001. (18)F-fluorodeoxyglucose positron emission tomography and MR imaging findings in Rasmussen encephalitis. AJNR Am. J. Neuroradiol. 22: 1291-1299. [Medline]

11. Hansen, A. E., McEvoy, F., Engelholm, S. A., Law, I. and Kristensen, A. T. 2011. FDG PET/CT imaging in canine cancer patients. Vet. Radiol. Ultrasound 52: 201-206. [Medline] [CrossRef]

12. Heo, J. H., Lee, S. R., Lee, S. T., Lee, K. M., Oh, J. H., Jang, D. P., Chang, K. T. and Cho, Z. H. 2011. Spatial distribution of glucose hypometabolism induced by intracerebroventricular streptozotocin in monkeys. J. Alzheimers Dis. 25: 517-523. [Medline]

13. Herholz, K., Coope, D. and Jackson, A. 2007. Metabolic and molecular imaging in neuro-oncology. Lancet Neurol. 6: 711724. [Medline] [CrossRef]

14. Irimajiri, M., Miller, M. A., Green, M. A., Jaeger, C. B., Luescher, A. U. and Hutchins, G. D. 2010. Cerebral metabolism in dogs assessed by (18)F-FDG PET: a pilot study to understand physiological changes in behavioral disorders in dogs. J. Vet. Med. Sci. 72: 1-6. [Medline] [CrossRef]

15. Jacobs, A. H., Kracht, L. W., Gossmann, A., Rüger, M. A., Thomas, A. V., Thiel, A. and Herholz, K. 2005. Imaging in neurooncology. NeuroRx 2: 333-347. [Medline] [CrossRef]

16. Juweid, M. E. and Cheson, B. D. 2006. Positron-emission tomography and assessment of cancer therapy. N. Engl. J. Med. 354: 496-507. [Medline] [CrossRef]

17. Kang, B. T., Kim, S. G., Lim, C. Y., Gu, S. H., Jang, D. P., Kim, Y. B., Kim, D. Y., Woo, E. J., Cho, Z. H. and Park, H. M. 2010. Correlation between fluorodeoxyglucose positron emission tomography and magnetic resonance imaging findings of non-suppurative meningoencephalitis in 5 dogs. Can. Vet. J. 51: 986-992. [Medline]

18. Kang, B. T., Ko, K. J., Jang, D. P., Han, J. Y., Lim, C. Y., Park, C., Yoo, J. H., Kim, J. W., Jung, D. I., Kim, Y. B., Woo, E. J., Cho, Z. H. and Park, H. M. 2009. Magnetic resonance imaging of the canine brain at 7 T. Vet. Radiol. Ultrasound 50: 615-621. [Medline] [CrossRef] 
19. Kang, B. T., Park, C., Yoo, J. H., Gu, S. H., Jang, D. P., Kim, Y. B., Woo, E. J., Kim, D. Y., Cho, Z. H. and Park, H. M. 2009. 18F-fluorodeoxyglucose positron emission tomography and magnetic resonance imaging findings of primary intracranial histiocytic sarcoma in a dog. J. Vet. Med. Sci. 71: 1397-1401. [Medline] [CrossRef]

20. Keyes, J. W. Jr. 1995. SUV: standard uptake or silly useless value? J. Nucl. Med. 36: 1836-1839. [Medline]

21. Kim, M. J., Jeon, H. A., Lee, K. M., Son, Y. D., Kim, Y. B. and Cho, Z. H. 2009. Neuroimaging features in a case of developmental central auditory processing disorder. J. Neurol. Sci. 277: 176-180. [Medline] [CrossRef]

22. Lawrence, J., Rohren, E. and Provenzale, J. 2010. PET/CT today and tomorrow in veterinary cancer diagnosis and monitoring: fundamentals, early results and future perspectives. Vet. Comp. Oncol. 8: 163-187. [Medline]

23. LeBanc, A. K., Jakoby, B., Townsend, D. W. and Daniel, G. B. 2008. Thoracic and abdominal organ uptake of 2-deoxy-2-[18F] fluoro-D-glucose (18FDG) with positron emission tomography in the normal dog. Vet. Radiol. Ultrasound 49: 182-188. [Medline] [CrossRef]

24. Lee, M. S., Ko, J., Lee, A. R., Lee, I. H., Jung, M. A., Austin, B., Chung, H., Nahm, S. and Eom, K. 2010. Effects of anesthetic protocol on normal canine brain uptake of $18 \mathrm{~F}-\mathrm{FDG}$ assessed by PET/CT. Vet. Radiol. Ultrasound 51: 130-135. [Medline]

25. Lee, B. Y., Newberg, A. B., Liebeskind, D. S., Kung, J. and Alavi, A. 2004. FDG-PET findings in patients with suspected encephalitis. Clin. Nucl. Med. 29: 620-625. [Medline] [CrossRef]

26. Leigh, E. J., Mackillop, E., Robertson, I. D. and Hudson, L. C. 2008. Clinical anatomy of the canine brain using magnetic reso- nance imaging. Vet. Radiol. Ultrasound 49: 113-121. [Medline] [CrossRef]

27. Martín-Vaquero, P., Da Costa, R. C., Echandi, R. L., Tosti, C. L., Knopp, M. V. and Sammet, S. 2011. Magnetic resonance imaging of the canine brain at 3 and 7 T. Vet. Radiol. Ultrasound 52: 25-32. [Medline]

28. Miletich, R. S. 2009. Positron emission tomography for neurologists. Neurol. Clin. 27: 61-88. [Medline] [CrossRef]

29. Phelps, M. E. 2000. Positron emission tomography provides molecular imaging of biological processes. Proc. Natl. Acad. Sci. U.S.A. 97: 9226-9233. [Medline] [CrossRef]

30. Sokoloff, L. 1986. Cerebral circulation, energy metabolism, and protein synthesis: general characteristics and principles of measurement, pp. 1-71. In: Positron Emission Tomography and Autoradiography, Principles and Applications for the Brain and Heart, Raven press, New York.

31. Son, Y. D., Cho, Z. H., Kim, H. K., Choi, E. J., Lee, S. Y., Chi, J. G., Park, C. W. and Kim, Y. B. 2012. Glucose metabolism of the midline nuclei raphe in the brainstem observed by PET-MRI fusion imaging. Neuroimage 59: 1094-1097. [Medline] [CrossRef]

32. West, J., Fitzpatrick, J. M., Wang, M. Y., Dawant, B. M., Maurer, C. R. Jr., Kessler, R. M., Maciunas, R. J., Barillot, C., Lemoine, D., Collignon, A., Maes, F., Suetens, P., Vandermeulen, D., van den Elsen, P. A., Napel, S., Sumanaweera, T. S., Harkness, B., Hemler, P. F., Hill, D. L., Hawkes, D. J., Studholme, C., Maintz, J. B., Viergever, M. A., Malandain, G. and Woods, R. P. 1997. Comparison and evaluation of retrospective intermodality brain image registration techniques. J. Comput. Assist. Tomogr. 21: 554-566. [Medline] [CrossRef] 